\title{
AGS BOOSTER BEAM POSITION, TUNE, AND LONGITUDINAL PROFILE DATA ACQUISITION SYSTEM*
}

\author{
K.A.Brown ${ }^{\dagger}$, L.Ahrens, F.Severino, K.Smith, M.Wilinski, \\ C-AD Dept., BNL, Upton, NY
}

\section{Abstract}

In this paper we will describe a data acquisition system designed and developed for the AGS Booster. The system was motivated by the need to get high quality beam diagnostics from the AGS Booster. This was accomplished by locating the electronics and digital data acquisition close to the Booster ring, to minimize loss of bandwidth in the original signals. In addition we had to develop the system rapidly and at a low cost. The system consists of a Lecroy digital oscilloscope which is interfaced through a National Instruments LabView ${ }^{\mathrm{TM}}$ server application, developed for this project. This allows multiple client applications to time share the scope without interfering with each other. We will present a description of the system design along with example clients that we have implemented.

\section{INTRODUCTION}

The AGS Booster has operated since 1991 as a preinjector of protons and heavy ions into the AGS. Over the past decade the operation of the Booster has evolved such that it now always operates in the linear regions of the magnetic elements. Most recently, though, the Booster was modified to allow for slow extraction of ions to the NASA Space Radiation Laboratory (NSRL). This facility is designed to employ heavy ion beams of many different ion species and at beam energies ranging from 0.04 to 3.07 $\mathrm{GeV} /$ nucleon. Many of the modes of operation required by NSRL will be operating the Booster at the limits of the magnets and respective power supplies. In order to allow operating at the highest fields it was necessary to upgrade the tune quadrupole trim power supplies, to provide a larger area of tune space maneuverability. To allow us to experimentally explore these high rigidity regions of the Booster lattice we needed a flexible yet powerful beam diagnostics system, which would provide the highest possible fidelity of information from Beam Position Monitors (BPM) and the Booster wall current monitor. In this report we will describe this system and show examples of its use.

\section{SYSTEM DESCRIPTION AND DESIGN}

Figure 1 shows a block diagram of the data acquisition system. The most fundamental component is a Lecroy Waverunner ${ }^{\mathrm{TM}}$ digital oscilloscope. This scope is capable of operating at $1 \mathrm{GSample} / \mathrm{sec}$, with $2 \mathrm{MSamples} / \mathrm{channel}$

\footnotetext{
${ }^{*}$ Work performed under Contract Number DE-AC02-98CH10886 with the auspices of the US Department of Energy.

† Email: kbrown@bnl.gov
}

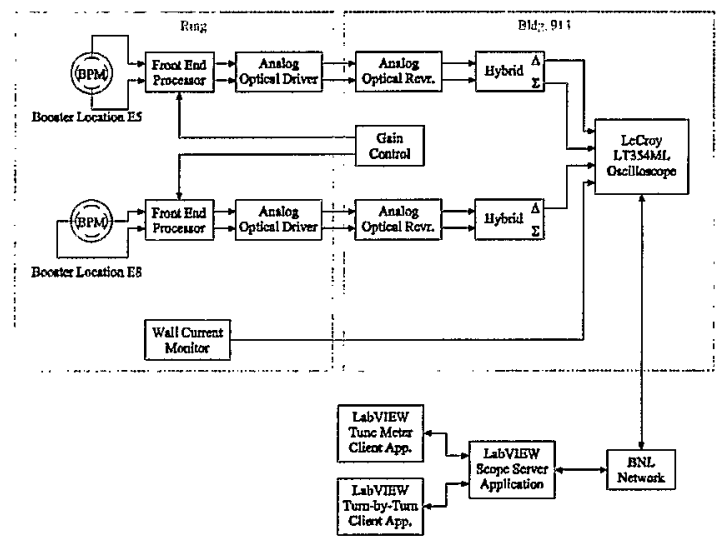

Figure 1: Booster BPM Data Acquisition Block Diagram.

memory depth. This allows sampling the BPM or Wall current monitor signals for as long as $2 \mathrm{msec}$ at $1 \mathrm{nsec}$ resolution. In practice we need only to sample up to $500 \mu \mathrm{sec}$ to get good resolution in the betatron tunes.

\section{Hardware Components}

The Booster BPM system has been described previously in $[1,2]$. Booster BPM's are located in $\beta_{m a x}$ locations in the Booster lattice. The system bandwidth range is from 0.05 to $30 \mathrm{MHz}$. The Booster has two Wall current monitors, one for RF phase loop operation and a second one for diagnostics. The Wall current monitor bandwidth is in the range from about $1 \mathrm{MHz}$ to $1 \mathrm{GHz}$. The Booster BPM's have the main electronics in the Booster tunnel and use optical fiber links to transmit data to electronics outside the tunnel. The BPM signals are then sent to an RF Hybrid, used as a combiner, to create sum and difference signals. The vertical sum and difference and the horizontal sum are input to the scope. The Wall current monitor signals are connected through high frequency coaxial cables directly to the Booster Main RF controls station. The digital oscilloscope we employ is located in a rack with the Booster Main RF controls.

\section{Software Components}

One of the advantages of using the Lecroy digital oscilloscope is the availability of driver modules and interface libraries through the use of National Instruments LabView $^{\mathrm{TM}}$ development environment. Since this system is basically a copy of an existing AGS application [2], we were able to adapt the existing LabView application code 
to the new scope and Booster parameters. But we didn't just make a copy of the AGS system. One of the limitations of the AGS system is the application's interface to an oscilloscope directly through a GPIB address. Only one application can communicate and control the scope during a measurement session. To enable the scope to be accessible to multiple simultaneous applications, we built a LabView server and adapted the applications to communicate with the scope through the server. Figure 2 shows the main window for the server application.

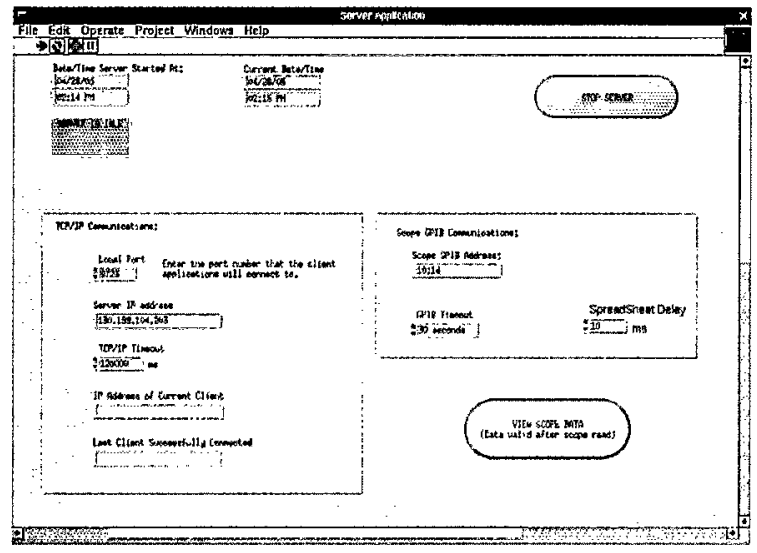

Figure 2: Server Main Window.

Tune Meter Client Even though the tune meter client was adapted from the AGS tune meter, there was still a substantial amount of development required to get the software to work with the new system. The most significant difference in the two systems is the amount of preprocessing that is done in the hardware. For the AGS tune meter system the BPM signals are heavily integrated, such that position information is retained over the period of one turn. The scope, in the AGS case, is clocked on the AGS revolution frequency, so that one sample is taken per revolution. This works well for obtaining a frequency from an FFT/DFT, but limits the amount of information that can be obtained from the BPM signal. In the new system we minimize the amount of processing outside the scope and sample the BPM signals (sum and difference signals) using the scopes internal clock.

In order to perform the same FFT/DFT operation on the data, we have to generate a position per turn array internally and feed that data to the FFT/DFT modules. To do this we have taken advantage of the ability to connect our own code to the LabView application, using the LabView CIN interface. The CIN interface is a mechanism provided by LabView for interfacing external c-code to the application. This allows us to take the BPM sum and difference arrays and use our own algorithms to generate the position/turn. As a benefit of this process we can provide any other information derived from the BPM signals, including integrated intensity, revolution frequency, and bunch length arrays. The problem of generating the position/turn data requires that we know a few things in advance, such as the RF frequency, the harmonic, and the expected bunch length. This information doesn't have to be exact, but good enough to allow the algorithm to have reasonable starting points. The algorithm begins by looking at just the sum data, to derive the locations and widths of the bunches.

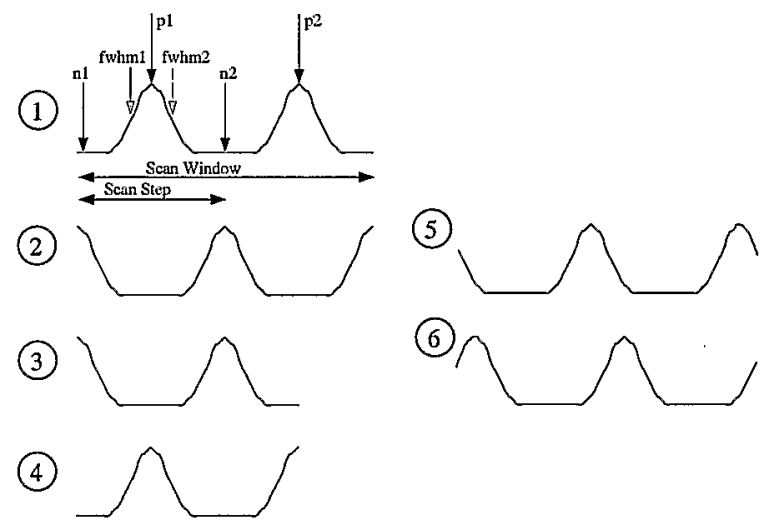

Figure 3: Bunch processing cases.

In figure 3 six cases that must be handled are shown. Since the scope trigger is not synchronous to the bunches, the start of a sample could occur on a bunch or someplace between two bunches. The algorithm cannot assume the data starts in a bunch free region of time. In the figure, case 1 is annotated to illustrate the events and sample windows used in the algorithm. In cases 2,5 , and 6 , the data begins on top of a bunch. For these cases the start point must be moved to just after the first partial bunch. In cases 3 and 4 the user defined frequency was set to high and the scan window isn't long enough to contain two full bunches. The user defined frequency cannot be set too low, either, since this risks bunches being interpreted as noise. The algorithm uses a multi-search approach that is adaptive and learns the proper parameters to use as it moves along the bunch train. The first task it must accomplish is to determine where to start and how wide a sample window to use. To accomplish this it uses a user supplied starting frequency and harmonic to set the window to contain two bunches worth of data. It must then search through the first window of data and set a starting point in the region between two bunches. Since it is possible that the signal to noise ratio could be small, especially given $1 \mathrm{nsec}$ sampling, the algorithm performs various permutations to the original data to enhance the bunch data. It does this in an adaptive way, applying only as much filtering and integration as required. To find a bunch the sum data is differentiated to obtain positive and negative slopes of the bunch. This allows determination of whether an edge is rising or falling. Once the starting point is determined the algorithm searches over a sample window of data to find two maximums (labeled $\mathrm{p} 1$ and $\mathrm{p} 2$ in the figure), separated approximately by a distance determined from the user supplied RF frequency, with each located in the region between a rising and falling edge. The algorithm makes various tests to determine that it is finding reason- 
able values. Based on the values of $\mathrm{p} 1$ and $\mathrm{p} 2$ it searches though the regions between the bunches to find the middle point between the bunches ( $\mathrm{n} 1$ and $\mathrm{n} 2$ in the figure). The algorithm then steps a distance of one Scan Step and repeats the exercise. It scans across the entire data set in this manner. In a second stage the algorithm searches around each maximum for full width half maximum points (fwhml and fwhm 2 in the figure) and refines the values for the bunch peak locations and the middle points between bunches. Finally, using the knowledge of the bunch positions it scans across the difference data to generate the position/turn array, using the integral of the area between the two fwhm points. The algorithm always adjusts parameters such that it can work its way into case 1 .

Turn by Turn Mountain Range Client As with the tune meter application, a LabView application exists for a turn by turn mountain range. In this case the adaption to the new system was more straight forward, since the only change that was needed was to have the application communicate with the server instead of directly to the scope. In fact, the existing AGS application can be used to communicate directly to the scope by simply giving it the correct GPIB/TCPIP address. This application takes a frequency, a harmonic, and an f-dot as variables and produces a mountain range by sampling the wall monitor at the required frequency to get the desired sample size.

\section{RESULTS}

Figure 4 shows the data analysis results window of the Tune Meter Client. The beam position/turn is shown above the Fourier transforms for the horizontal and vertical planes. Below the Fourier transforms is a frame for each plane that allows zooming in on the spectrum data.

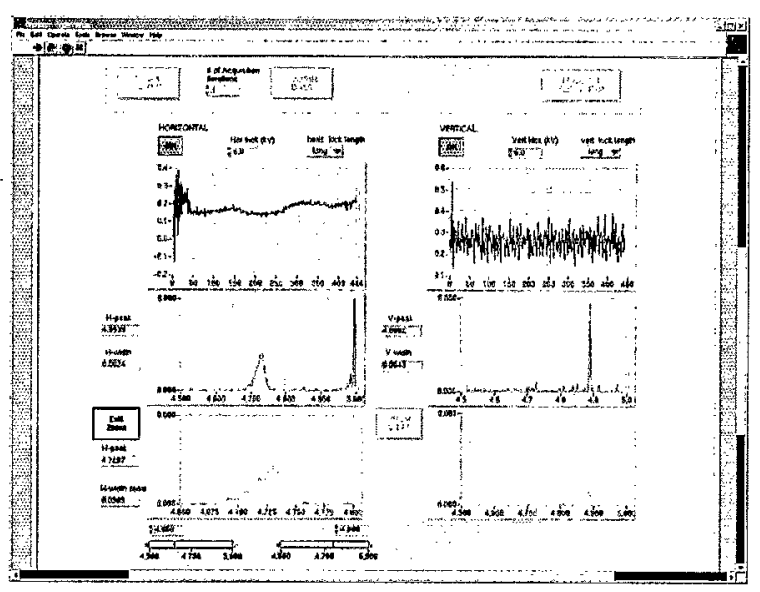

Figure 4: Booster Tune Meter Client.

Figure 5 shows the main window of the Turn by Turn Mountain Range Client. In this case, although the harmonic is 1 , two turns of the Wall current monitor are shown per trace. The data shows beam debunching as the accelerating $\mathrm{RF}$ is turned off on a zero B-dot porch.

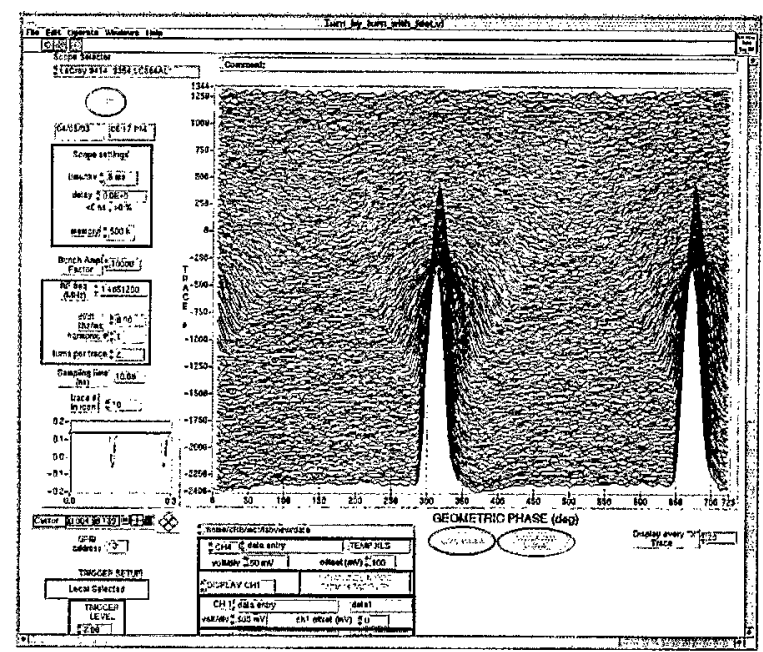

Figure 5: Booster Turn by Turn mountain range client.

\section{FUTURE DEVELOPMENTS}

By using a client/server communications architecture we have given ourselves the ability to provide a more general platform for beam diagnostics. To offer more flexibility to the system we are looking into providing a high frequency multiplex, to allow connecting different signals to the digital scope. This would allow selection of which BPM's to view, as well as development of new applications. We are also looking at being able to process the BPM data using fittings. In this case one would want to solve equation 1 [3].

$$
X_{n}=C+D n+A e^{\frac{(-n \pi \Delta Q)}{2}} \cos \left(2 \pi n\left(Q_{0}+\frac{n \delta Q}{2}\right)+\phi\right)
$$

Where $C, D, A, Q_{0}, \phi, \Delta Q$, and $\delta Q$, are, respectively; the position of the closed orbit at the BPM; the change in the closed orbit position per turn; the amplitude, tune, and phase of the betatron oscillations; the tune spread of the beam; and the tune shift per turn. $\mathrm{n}$ represents the turn number and $X_{n}$ is the position per turn.

\section{REFERENCES}

[1] D.J.Ciardullo et al, "Design of the AGS Booster Beam Position Monitor Electronics”, PAC'91,May 6-9, 1991, San Francisco, CA, p. 1431.

http://accelconf.web.cern.ch/AccelConf/p91/INDEX.HTM

[2] W.K.van Asselt et al, "The Tune Meter Systems at the AGS Complex", PAC'91, May 6-9, 1991, San Francisco, CA, ,p.1273

http://accelconf.web.cern.ch/AccelConf/p91/INDEX.HTM

[3] C.Gardner et al, "Turn-by-Turn Analysis of Proton and Gold Beams at Injection in the AGS booster", PAC'99,March 29April 2, 1999, New York, NY, p.2063 http://accelconf.web.cern.ch/AccelConf/p99/PROCS.HTM 